\title{
Design of Nonrecursive Digital Filters Using the Ultraspherical Window Function
}

\author{
Stuart W. A. Bergen \\ Department of Electrical and Computer Engineering, University of Victoria, P.O. Box 3055, STN CSC, Victoria, BC, Canada V8W $3 P 6$ \\ Email: sbergen@ece.uvic.ca
}

Andreas Antoniou

Department of Electrical and Computer Engineering, University of Victoria, P.O. Box 3055, STN CSC, Victoria, BC, Canada V8W $3 P 6$ Email:aantoniou@ieee.org

Received 13 September 2004; Revised 8 February 2005; Recommended for Publication by Ulrich Heute

\begin{abstract}
An efficient method for the design of nonrecursive digital filters using the ultraspherical window function is proposed. Economies in computation are achieved in two ways. First, through an efficient formulation of the window coefficients, the amount of computation required is reduced to a small fraction of that required by standard methods. Second, the filter length and the independent window parameters that would be required to achieve prescribed specifications in lowpass, highpass, bandpass, and bandstop filters as well as in digital differentiators and Hilbert transformers are efficiently determined through empirical formulas. Experimental results demonstrate that in many cases the ultraspherical window yields a lower-order filter relative to designs obtained using windows like the Kaiser, Dolph-Chebyshev, and Saramäki windows. Alternatively, for a fixed filter length, the ultraspherical window yields reduced passband ripple and increased stopband attenuation relative to those produced when using the alternative windows.
\end{abstract}

Keywords and phrases: nonrecursive digital filters, FIR filters, window functions, ultraspherical window, digital differentiators, Hilbert transformers.

\section{INTRODUCTION}

Window functions (or windows for short) are time-domain weighting functions that have found widespread usage in signal processing applications such as power spectral estimation, beamforming, and digital filter design. Windows can be categorized as fixed or adjustable [1]. Fixed windows have only one independent parameter, namely, the window length which controls the window's mainlobe width. Adjustable windows have two or more independent parameters, namely, the window length, as in fixed windows, and one or more additional parameters that can control other window characteristics. Each of the adjustable windows has been derived by exploiting certain characteristics of well-known polynomials to satisfy a particular criterion. For instance, the Kaiser and Saramäki windows [2,3] have two parameters and yield close approximations to discrete prolate functions, which have maximum energy concentration in the mainlobe. The Dolph-Chebyshev window [4] has two parameters and produces the minimum mainlobe width for a specified maximum sidelobe amplitude. The Kaiser, Saramäki, and DolphChebyshev windows can control the amplitude of the sidelobes relative to that of the mainlobe. The ultraspherical window $[5,6,7,8]$ has three parameters and through the proper choice of these parameters, the amplitude of the sidelobes relative to that of the mainlobe can be controlled as in the Kaiser, Saramäki, and Dolph-Chebyshev windows, and, in addition, different sidelobe patterns can be achieved. With the judicious selection of the ultraspherical window's additional parameter, a unique family of sidelobe patterns, which includes both the Dolph-Chebyshev and Saramäki patterns as special cases, can be readily obtained by generating the window's coefficients through a closed-form solution $[5,7]$. Furthermore, a comparison with other windows has shown that a difference exists in performance between the ultraspherical and Kaiser windows, which depends critically on the set of prescribed spectral characteristics [8]. In [6] Deczky used the ultraspherical window to provide a proof-of-concept example for nonrecursive filter design. In [9] Johnson and Johnson used ultraspherical polynomials for the approximation problem in analog filter design.

The window method for nonrecursive digital filter design is based largely on closed-form solutions [10]. As a result, it is straightforward to apply and entails a relatively insignificant amount of computation. Unfortunately, the window method usually yields suboptimal designs whereby the filter 
order required to satisfy a given set of specifications is not the lowest that can be achieved. On the other hand, multivariable optimization algorithms for nonrecursive digital-filter design, for example, the weighted-Chebyshev method of Parks and McClellan [11, 12] and the more recent generalized Remez method of Shpak and Antoniou [13] can yield optimal designs with respect to some error criterion; however, these algorithms generally require a large amount of computation and are, therefore, unsuitable for real- or quasi-realtime applications like portable multimedia devices where onthe-fly designs that adapt to changing environmental conditions such as battery power and quality-of-service issues are required. Simple signal processing algorithms and structures [14] can address these problems by trading between the accuracy of results and the utilization of implementation resources. In $[15,16]$ a window-based algorithmic approach to the design of low-power frequency-selective digital filters is presented whereby reduction of the average power consumption of the filter is achieved in speech processing and high-fidelity hardware by dynamically varying the filter length based on signal statistics. In applications such as these, flexible windows that would satisfy prescribed filter specifications and whose coefficients can be generated quickly are highly desirable.

In this paper, an efficient formulation for generating the coefficients of the ultraspherical window is proposed and its application for the design of nonrecursive digital filters, digital differentiators, and Hilbert transformers that would satisfy prescribed specifications is demonstrated. The paper is structured as follows. Section 2 introduces relevant information concerning the ultraspherical window. Section 3 describes an efficient formulation for generating the coefficients of the ultraspherical window. Section 4 deals with the design of nonrecursive digital filters using the ultraspherical window and provides comparisons with designs based on other windows as well as designs based on the Remez algorithm. Section 5 deals with the design of digital differentiators and Hilbert transformers that would satisfy prescribed specifications. Section 6 provides design examples. Section 7 provides concluding remarks.

\section{THE ULTRASPHERICAL WINDOW}

The coefficients of a right-sided ultraspherical window can be calculated explicitly as $[5,7]$

$$
\begin{aligned}
w(n T)= & \frac{A}{p-n}\left(\begin{array}{c}
\mu+p-n-1 \\
p-n-1
\end{array}\right) \\
& \cdot \sum_{m=0}^{n}\left(\begin{array}{c}
\mu+n-1 \\
n-m
\end{array}\right)\left(\begin{array}{c}
p-n \\
m
\end{array}\right) B^{m} \text { for } n=0,1, \ldots, N-1,
\end{aligned}
$$

where [17]

$$
\left(\begin{array}{l}
\alpha \\
p
\end{array}\right)=\frac{\alpha(\alpha-1) \cdots(\alpha-p+1)}{p !} \quad \text { for } p \geq 1
$$

with $\left(\begin{array}{l}\alpha \\ 0\end{array}\right)=\left(\begin{array}{l}\alpha \\ \alpha\end{array}\right)=1$ because $\left(\begin{array}{l}n \\ k\end{array}\right)=\left(\begin{array}{c}n \\ n-k\end{array}\right) \cdot T$ is the interval between samples, and

$$
\begin{aligned}
A & = \begin{cases}\mu x_{\mu}^{p} & \text { for } \mu \neq 0, \\
x_{\mu}^{p} & \text { for } \mu=0,\end{cases} \\
B & =1-x_{\mu}^{-2}, \\
p & =N-1,
\end{aligned}
$$

where $N$ may be odd or even. In $(1), \mu, x_{\mu}$, and $N$ are independent parameters and $w[(N-n-1) T]=w(n T)$, that is, the window is symmetrical. A normalized window is obtained as $\widehat{w}(n T)=w(n T) / w(L T)$ where

$$
L= \begin{cases}\frac{N-1}{2} & \text { for odd } N \\ \frac{N-2}{2} & \text { for even } N\end{cases}
$$

The independent parameter $x_{\mu}$ can be expressed as

$$
x_{\mu}=\frac{x_{N-1}^{(\mu)}}{\cos (\beta \pi / N)},
$$

where $\beta \geq 1$ and $x_{N-1}^{(\mu)}$ is the largest zero of the ultraspherical polynomial $C_{N-1}^{\mu}(x)$ [17], which can be found using Algorithm 1 in [8]. The new independent parameter $\beta$ in (7) is the so-called shape parameter and can be used to set the null-to-null width of a window to $4 \beta \pi / N$,that is, $\beta$ times that of the rectangular window [3].

The Dolph-Chebyshev and Saramäki windows are special cases of the ultraspherical window and can be obtained by letting $\mu=0$ and 1 , respectively, in (1). Another special case of interest is when $\mu=1 / 2$, which produces windows based on the Legendre polynomial. Figure 1 shows the normalized amplitude spectrum for the ultraspherical window of length $N=51$ designed with $\beta=2$ and $\mu=-0.5,0$, and 1. A detailed description of the ultraspherical window's properties and its relation to other windows can be found in [8].

\section{EFFICIENT FORMULATION FOR WINDOW COEFFICIENTS}

A reduction in the computational complexity associated with the window method can be achieved by reducing the amount of computation required to generate the window coefficients. For the ultraspherical window, the primary computational bottleneck in (1) is due to the recursive evaluation of the binomial coefficients using (2). In its current form, (1) requires the evaluation of $(L+1)+\sum_{n=0}^{L} \sum_{m=0}^{n} 2=L^{2}+4 L+3$ binomial coefficients where $L$ is given by (6). By exploiting certain redundancies in (1), the number of binomial-coefficient evaluations can be reduced quite significantly and the computational complexity associated with the ultraspherical window can be reduced. To begin with, the first binomial-coefficient 


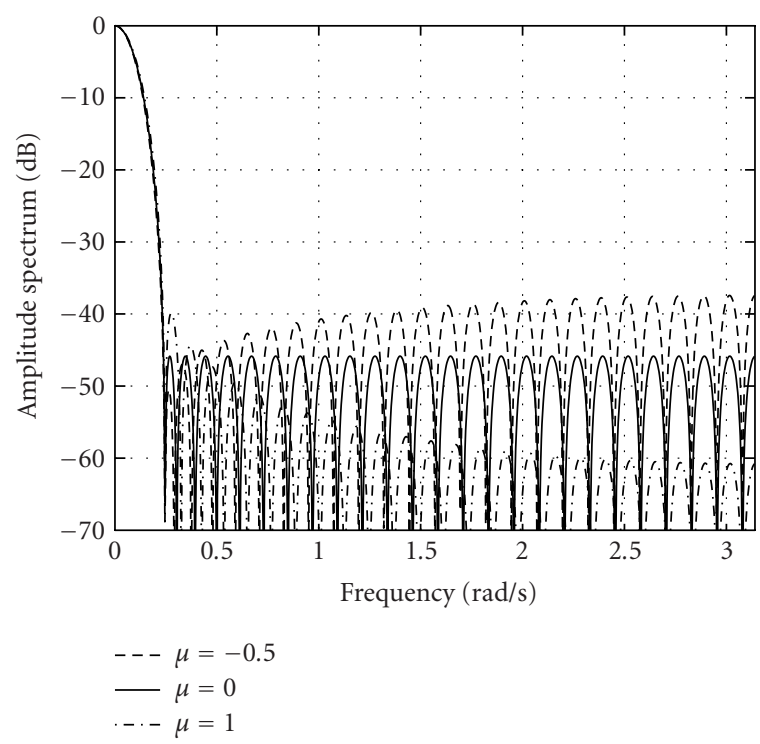

FIGURE 1: Normalized amplitude spectrum for the ultraspherical window of length $N=51$ designed with $\beta=2$ and $\mu=-0.5$ (dashed line), 0 (solid line), and 1 (dashed-dotted line).

expression in (1) can be expressed as

$$
v_{0}(n)=\left(\begin{array}{c}
\mu+p-n-1 \\
p-n-1
\end{array}\right)=\left(\begin{array}{c}
\alpha_{0}-n \\
p_{0}-n
\end{array}\right),
$$

where $\alpha_{0}=\mu+p-1$ and $p_{0}=p-1$. Using the identity [17]

$$
\left(\begin{array}{l}
a \\
b
\end{array}\right)=\frac{a !}{(a-b) ! b !}
$$

$v_{0}(n)$ can be represented as

$$
\begin{aligned}
v_{0}(n)= & \frac{\left(\alpha_{0}-n\right) !}{\left(\alpha_{0}-p_{0}\right) !\left(p_{0}-n\right) !} \\
= & \frac{p_{0}-n+1}{\alpha_{0}-n+1} \cdot \frac{p_{0}-n+2}{\alpha_{0}-n+2} \cdots \frac{p_{0}-1}{\alpha_{0}-1} \\
& \cdot \frac{p_{0}}{\alpha_{0}} \cdot\left(\begin{array}{c}
\alpha_{0} \\
p_{0}
\end{array}\right) \quad \text { for } n \geq 1
\end{aligned}
$$

which leads to the recurrence relationships

$v_{0}(0)=\left(\begin{array}{l}\alpha_{0} \\ p_{0}\end{array}\right), \quad v_{0}(n)=\frac{p_{0}-n+1}{\alpha_{0}-n+1} v_{0}(n-1) \quad$ for $n \geq 1$.

In this formulation, the evaluation of one binomial coefficient replaces the evaluation of $L+1$ binomial coefficients thereby providing a savings of $L$ binomial-coefficient evaluations.

Next, we express the second binomial-coefficient expression in (1) as

$$
v_{1}(n, m)=\left(\begin{array}{c}
\mu+n-1 \\
n-m
\end{array}\right)=\left(\begin{array}{c}
\alpha_{1} \\
g
\end{array}\right),
$$

where $\alpha_{1}=\mu+n-1$ and $g=n-m$. Observing that $v_{1}(n, n)=$ $\left(\begin{array}{l}\alpha_{1} \\ 0\end{array}\right)=1$ and using the recursive identity [17]

$$
\left(\begin{array}{l}
a \\
0
\end{array}\right)=1, \quad\left(\begin{array}{l}
a \\
b
\end{array}\right)=\frac{a-b+1}{b}\left(\begin{array}{c}
a \\
b-1
\end{array}\right) \quad \text { for } b \geq 1
$$

$v_{1}(n, m)$ can be represented as

$$
\begin{aligned}
v_{1}(n, m) & =\left(\begin{array}{c}
\alpha_{1} \\
g
\end{array}\right) \\
& =\frac{\alpha_{1}-g+1}{g} \cdot \frac{\alpha_{1}-g+2}{g-1} \cdots \frac{\alpha_{1}-1}{2} \cdot \alpha_{1} \cdot\left(\begin{array}{c}
\alpha_{1} \\
0
\end{array}\right) .
\end{aligned}
$$

This analysis leads to the recurrence relationships

$v_{1}(n, n)=1, \quad v_{1}(n, m)=\frac{\mu+m}{n-m} v_{1}(n, m+1) \quad$ for $0 \leq m<n$.

This formulation is equivalent to the evaluation of $L$ binomial coefficients replacing the evaluation requirements of $\sum_{n=0}^{L} \sum_{m=0}^{n} 1=(1 / 2) L^{2}+(3 / 2) L+1$ binomial coefficients, which would result in a savings of $(1 / 2) L^{2}+(1 / 2) L+1$ binomial-coefficient evaluations.

Finally, we express the third binomial-coefficient expression in (1) as

$$
v_{2}(n, m)=\left(\begin{array}{c}
p-n \\
m
\end{array}\right)=\left(\begin{array}{c}
\alpha_{2} \\
m
\end{array}\right)
$$

where $\alpha_{2}=p-n$. Observing that $v_{2}(n, 0)=\left(\begin{array}{c}\alpha_{2} \\ 0\end{array}\right)=1$ and using the recursive identity in $(13), v_{2}(n, m)$ can be represented as

$$
\begin{aligned}
v_{2}(n, m) & =\left(\begin{array}{c}
\alpha_{2} \\
m
\end{array}\right) \\
& =\frac{\alpha_{2}-m+1}{m} \cdot \frac{\alpha_{2}-m+2}{m-1} \cdots \frac{\alpha_{2}-1}{2} \cdot \alpha_{2} \cdot\left(\begin{array}{c}
\alpha_{2} \\
0
\end{array}\right) .
\end{aligned}
$$

This leads to the recurrence relationships

$$
\begin{aligned}
v_{2}(n, 0) & =1, \\
v_{2}(n, m) & =\frac{\alpha_{2}-m+1}{m} v_{2}(n, m-1) \quad \text { for } 1 \leq m \leq n .
\end{aligned}
$$

This formulation is equivalent to the evaluation of $L$ binomial coefficients replacing the evaluation of $\sum_{n=0}^{L} \sum_{m=0}^{n} 1=$ $(1 / 2) L^{2}+(3 / 2) L+1$ binomial coefficients, which provides a savings of $(1 / 2) L^{2}+(1 / 2) L+1$ binomial-coefficient evaluations. 


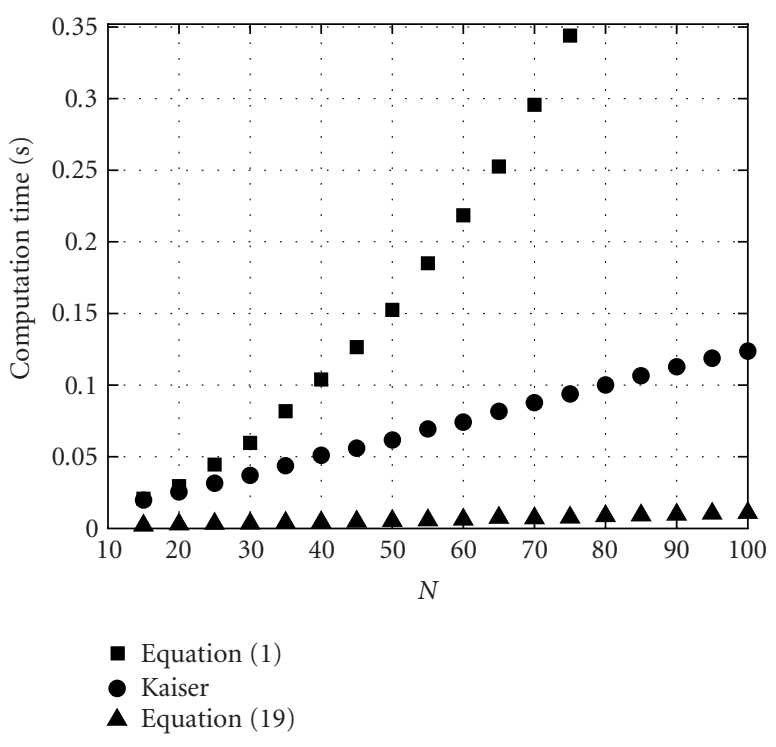

FIgURE 2: Computation time associated with (1) (squares), (19) (triangles), and for the Kaiser window (circles) versus the window length $N$.

Using the above expressions, the coefficients of the rightsided ultraspherical window of length $N$ can be calculated using the formulation

$$
\begin{aligned}
w(n T)= & \frac{A}{p-n} v_{0}(n) \\
& \cdot \sum_{m=0}^{n} v_{1}(n, m) v_{2}(n, m) B^{m} \quad \text { for } n=0,1, \ldots, N-1,
\end{aligned}
$$

where $v_{0}(n), v_{1}(n, m)$, and $v_{2}(n, m)$ are calculated using the recurrence relationships provided by (11), (15), and (18), respectively, and $A, B$, and $p$ are given by (3), (4), and (5), respectively. ${ }^{1}$ This method requires the recursive evaluation of $2 L+1$ binomial coefficients, which constitutes a computational complexity of $O(N)$ as compared with the evaluation of $L^{2}+4 L+3$ binomial coefficients required by (1), which constitutes a computational complexity of $O\left(N^{2}\right)$. In this way, an overall savings of $L^{2}+2 L+2$ binomial-coefficient evaluations can be achieved. Figure 2 shows the computation time required to evaluate the coefficients of the ultraspherical window using (1) and (19) versus the window length. ${ }^{2}$ The time to compute the coefficients of the Kaiser window is included for comparison. The zeroth-order modified Bessel function of the first kind $I_{0}(x)$ was evaluated to an accuracy of $\epsilon=10^{-10}$. As can be seen, the new formulation given by (19) provides a substantial computational savings over both the original formulation given by (1) and the formulation for generating the coefficients of the Kaiser window.

\footnotetext{
${ }^{1}$ An efficient MATLAB program for the computation of the window coefficients can be obtained from the authors.

${ }^{2}$ The computation time was measured using the MATLAB stopwatch commands tic and toc which return the total CPU time used to execute the code between the two commands.
}

\section{NONRECURSIVE DIGITAL FILTER DESIGN}

The window method produces filters with a symmetrical impulse response, thereby achieving constant group delay and filter realizations with a reduced number of multiplications [10]. Good comparisons that contrast various filters and their attributes can be found in $[10,18]$. In the window method, an idealized frequency response is assumed and upon the application of the Fourier series, an infiniteduration impulse response is obtained. For a lowpass filter, we have

$$
H_{i d}\left(e^{j \omega T}\right)= \begin{cases}1 & \text { for }|\omega| \leq \omega_{c}, \\ 0 & \text { for } \omega_{c}<|\omega| \leq \frac{\omega_{s}}{2},\end{cases}
$$

where $\omega_{c}$ and $\omega_{s}$ are the cutoff and sampling frequencies, respectively. The infinite-duration impulse response is obtained as

$$
h_{i d}(n T)= \begin{cases}\frac{\omega_{c}}{\pi} & \text { for } n=0, \\ \frac{1}{n \pi} \sin \omega_{c} n T & \text { for } n \neq 0,\end{cases}
$$

where $-\infty \leq n \leq \infty$. The design of highpass, bandpass, and bandstop filters is discussed later.

A realizable filter is obtained by multiplying the infiniteduration impulse response by the window function, that is, by letting

$$
h_{0}(n T)=w(n T) h_{i d}(n T)
$$

where $w(n T)$ is a window function of length $N=2 M+1$. If $N$ is odd, $M$ is an integer and $|n|=\{0,1,2, \ldots, M\}$ is used for both the window and impulse response. If $N$ is even, $M$ is a fraction and $|n|=\{0.5,1.5,2.5, \ldots, M\}$ is used [10]. Odd-length nonrecursive filters are assumed throughout this paper because the frequency response of an evenlength symmetric nonrecursive filter is 0 at the Nyquist frequency, which is inappropriate for highpass and bandstop filters. However, this property of even-length nonrecursive filters can be used for the design of Hilbert transformers as discussed in Section 5.2. A causal filter can be obtained by delaying the impulse response by a period $M T$, that is,

$$
h(n T)=h_{0}[(n-M) T] \quad \text { for } 0 \leq n \leq N-1 .
$$

The frequency response of the filter is given by the convolution of the idealized frequency response and the spectral representation of the window, that is,

$$
H\left(e^{j \omega T}\right)=\frac{1}{\omega_{s}} \int_{-\omega_{s} / 2}^{\omega_{s} / 2} H_{i d}\left(e^{j \theta T}\right) W\left(e^{j(\omega-\theta) T}\right) d \theta,
$$

where

$$
W\left(e^{j \omega T}\right)=\sum_{n=-M}^{M} w(n T) e^{-j \omega T} .
$$




\subsection{Choice of window parameters}

A nonrecursive (noncausal) lowpass filter is typically required to satisfy the equations

$$
\begin{array}{rlrl}
1-\delta_{p} & \leq H\left(e^{j \omega T}\right) \leq 1+\delta_{p} & & \text { for }|\omega| \in\left[0, \omega_{p}\right], \\
-\delta_{a} \leq H\left(e^{j \omega T}\right) & \leq \delta_{a} & \text { for }|\omega| \in\left[\omega_{a}, \frac{\omega_{s}}{2}\right],
\end{array}
$$

where $\delta_{p}$ and $\delta_{a}$ are the passband and stopband ripples and $\omega_{p}$ and $\omega_{a}$ are the passband and stopband edge frequencies, respectively. In nonrecursive filters designed using the window method, the passband ripple turns out to be approximately equal to the stopband ripple, that is, $\delta_{p} \approx \delta_{a}$. Therefore, one can design a filter that has a prescribed passband ripple or a prescribed stopband ripple. If the specifications call for a maximum passband ripple $A_{p}$ and a minimum stopband attenuation $A_{a}$, both specified in $\mathrm{dB}$, then it can easily be shown that [10]

$$
\delta_{p}=\frac{10^{0.05 A_{p}}-1}{10^{0.05 A_{p}}+1}, \quad \delta_{a}=10^{-0.05 A_{a}} .
$$

By designing a filter on the basis of

$$
\delta=\min \left(\delta_{p}, \delta_{a}\right),
$$

then if $\delta=\delta_{p}$, a filter will be obtained that has a passband ripple which is equal to $A_{p} \mathrm{~dB}$ and a minimum stopband attenuation which is greater than $A_{a} \mathrm{~dB}$; and if $\delta=\delta_{a}$, a filter will be obtained that has a minimum stopband attenuation which is equal to $A_{a} \mathrm{~dB}$ and a passband ripple which is less than $A_{p} \mathrm{~dB}$.

The ultraspherical window parameters $\mu, x_{\mu}$, and $N$ must be chosen such that the filter specifications are satisfied with the lowest possible filter length $N$. For a given set of prescribed specifications, the optimum values of $\mu$ and $x_{\mu}$ could be determined through a trial-and-error approach but such an approach would be laborious and time-consuming. Fortunately, a fairly general method that parallels Kaiser's method [2] can be used to design filters that satisfy arbitrary prescribed filter specifications. Through extensive experimentation, we found out that parameters $\mu$ and $x_{\mu}$ control the passband and stopband ripples and, consequently, the actual stopband attenuation, namely,

$$
A_{a}=-20 \log _{10}(\delta)
$$

Strictly speaking, parameter $x_{\mu}$ alters the window's ripple ratio at the expense of the null-to-null width, in effect, providing a tradeoff between the two just like parameter $\alpha$ in the Kaiser window [2] and parameter $x_{0}$ in the DolphChebyshev window [4]. Thus $x_{\mu}$ has a strong influence on the stopband attenuation. On the other hand, parameter $\mu$ controls the window's sidelobe pattern which affects the stopband attenuation but not to the extent that $x_{\mu}$ does. This property is observed in Figure 1 where windows with $\mu=0$ and 1 yield ripple ratios of -45.84 and $-39.85 \mathrm{~dB}$, respectively. On the other hand, the filter length $N$ controls the transition bandwidth of the filter, namely,

$$
B_{t}=\omega_{a}-\omega_{p}
$$

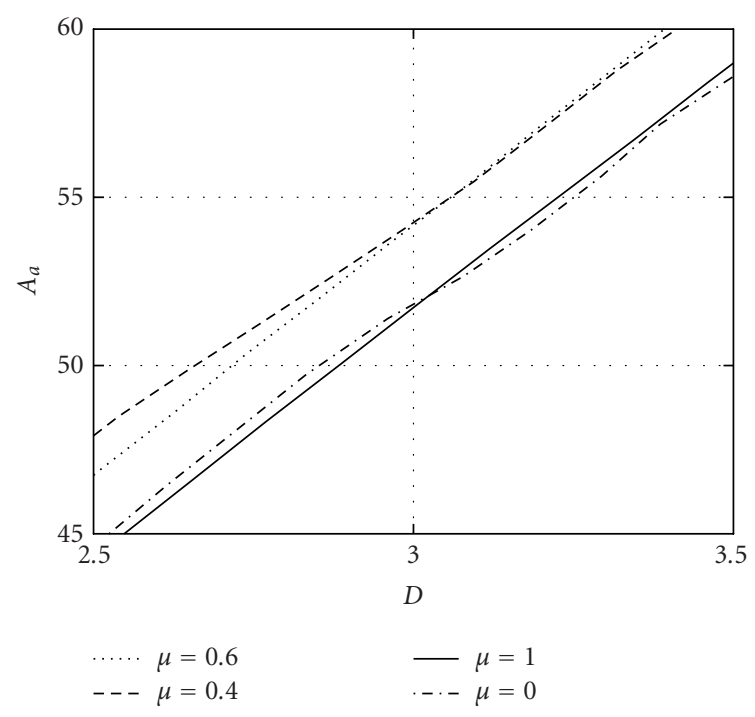

FIGURE 3: Stopband attenuation versus $D$ for filters designed using ultraspherical windows with $\mu=0$ (dash-dotted line), 0.4 (dashed line), 0.6 (dotted line), and 1 (solid line) for the filter design parameters $N=127, \omega_{c}=0.4 \pi \mathrm{rad} / \mathrm{s}$, and $\omega_{s}=2 \pi \mathrm{rad} / \mathrm{s}$.

but has little effect on the stopband attenuation. Consequently, the required value of $N$ is dependent on parameter $\mu$ while being relatively independent of parameter $x_{\mu}$.

The value of parameter $\mu$ that minimizes the filter length for a set of prescribed specifications can be determined by comparing the performance of filters designed using the ultraspherical window with varying values of the adjustable parameters for a fixed filter length and cutoff frequency as in [3]. The transition bandwidth is measured from the resulting filter and used to calculate the performance measure

$$
D=\frac{B_{t}(N-1)}{\omega_{s}}
$$

which is a normalized transition bandwidth that is approximately independent of the filter length $[2,3,19]$. Figure 3 shows plots of the stopband attenuation versus $D$ for filters designed using the ultraspherical window with $\mu=0,0.4$, 0.6 , and 1 . As can be seen, the filter performance depends critically on the choice of parameter $\mu$. In addition, we note that there is no unique fixed value of $\mu$ that yields minimum stopband attenuation, that is, the optimal value of $\mu$ changes with $D$. As such, it is possible to select an optimal value of $\mu$ that minimizes the filter length for a set of prescribed specifications. The value of $\mu$ that minimizes the filter length was found by calculating the value of $\mu$ that maximizes the stopband attenuation for a given normalized transition bandwidth $D$. Through curve fitting, an empirical formula for the optimal $\mu$ was derived as

$$
\mu=-1.721 \times 10^{-5} A_{a}^{2}+6.721 \times 10^{-3} A_{a}+1.897 \times 10^{-1} .
$$

This estimate provides relatively accurate predictions for $\mu$ for most practical purposes, that is, it holds true for low as well as high values of $N$. 


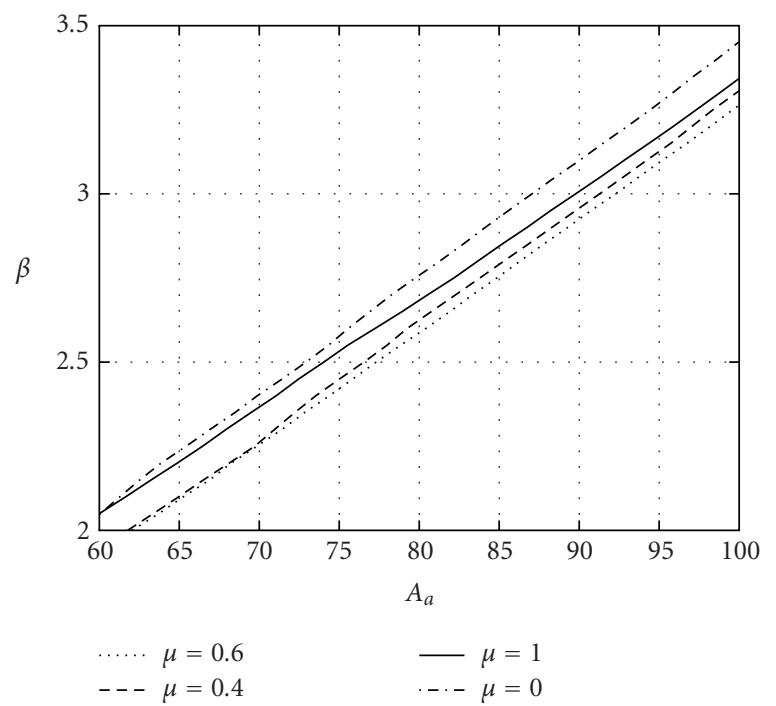

Figure 4: Parameter $\beta$ versus stopband attenuation for filters designed using ultraspherical windows with $\mu=0$ (dash-dotted line), 0.4 (dashed line), 0.6 (dotted line), and 1 (solid line) for the filter design parameters $N=127, \omega_{c}=0.4 \pi \mathrm{rad} / \mathrm{s}$, and $\omega_{s}=2 \pi \mathrm{rad} / \mathrm{s}$.

The minimum filter length required to achieve a desired stopband attenuation and transition bandwidth can be determined as the smallest odd integer satisfying the inequality [2]

$$
N \geq \frac{D \omega_{s}}{B_{t}}+1
$$

From (33) it becomes clear that $N$ can be predicted by obtaining an accurate approximation for $D$. As can be observed in Figure 3, $D$ is influenced by both the stopband attenuation and the parameter $\mu$. Through curve fitting, an empirical formula was deduced for $D$ corresponding to the value of $\mu$ given by (32) as

$$
D=\left\{\begin{array}{c}
4.645 \times 10^{-5} A_{a}^{2}+6.216 \times 10^{-2} A_{a} \\
-4.818 \times 10^{-1} \quad \text { for } A_{a} \leq 80, \\
1.710 \times 10^{-5} A_{a}^{2}+7.089 \times 10^{-2} A_{a} \\
-8.937 \times 10^{-1} \quad \text { for } A_{a}>80 .
\end{array}\right.
$$

The final window parameter $x_{\mu}$ provides a trade-off between the stopband attenuation and the transition bandwidth of the filter and can be determined using (7). It is clear that parameter $x_{\mu}$ can be predicted by obtaining an approximation for parameter $\beta$. Figure 4 shows plots of parameter $\beta$ versus stopband attenuation for filters designed using the ultraspherical window with $\mu=0,0.4,0.6$, and 1 . As can be seen, $\beta$ varies significantly depending on the choice of the stopband attenuation and parameter $\mu$. Through curve fitting, an empirical formula was derived for parameter $\beta$,
TABLE 1: Model coefficients for parameter $D$.

\begin{tabular}{cccc}
\hline$\mu$ & $a$ & $b$ & $c$ \\
\hline 0.0 & $-4.198 \mathrm{E}-5$ & $7.784 \mathrm{E}-2$ & $-7.778 \mathrm{E}-1$ \\
0.1 & $-2.961 \mathrm{E}-5$ & $7.574 \mathrm{E}-2$ & $-7.659 \mathrm{E}-1$ \\
0.2 & $-1.747 \mathrm{E}-5$ & $7.348 \mathrm{E}-2$ & $-7.369 \mathrm{E}-1$ \\
0.3 & $-5.808 \mathrm{E}-6$ & $7.109 \mathrm{E}-2$ & $-6.924 \mathrm{E}-1$ \\
0.4 & $6.462 \mathrm{E}-6$ & $6.844 \mathrm{E}-2$ & $-6.266 \mathrm{E}-1$ \\
0.5 & $3.221 \mathrm{E}-5$ & $6.408 \mathrm{E}-2$ & $-5.048 \mathrm{E}-1$ \\
0.6 & $6.111 \mathrm{E}-5$ & $5.957 \mathrm{E}-2$ & $-3.733 \mathrm{E}-1$ \\
0.7 & $7.789 \mathrm{E}-5$ & $5.736 \mathrm{E}-2$ & $-3.061 \mathrm{E}-1$ \\
0.8 & $6.328 \mathrm{E}-5$ & $5.975 \mathrm{E}-2$ & $-3.531 \mathrm{E}-1$ \\
0.9 & $3.620 \mathrm{E}-5$ & $6.391 \mathrm{E}-2$ & $-4.377 \mathrm{E}-1$ \\
1.0 & $1.532 \mathrm{E}-5$ & $6.717 \mathrm{E}-2$ & $-4.974 \mathrm{E}-1$ \\
\hline
\end{tabular}

which corresponds to the value of $\mu$ given by (32), as

$$
\beta=\left\{\begin{aligned}
4.024 & \times 10^{-5} A_{a}^{2}+2.423 \times 10^{-2} A_{a} \\
& +3.574 \times 10^{-1} \quad \text { for } A_{a} \leq 60 \\
7.303 & \times 10^{-5} A_{a}^{2}+2.079 \times 10^{-2} A_{a} \\
& +4.447 \times 10^{-1} \quad \text { for } 60<A_{a} \leq 120 \\
6.733 & \times 10^{-6} A_{a}^{2}+3.337 \times 10^{-2} A_{a} \\
& -1.192 \times 10^{-1} \quad \text { for } 120<A_{a} \leq 180 .
\end{aligned}\right.
$$

Equations (32), (33), (34), and (35) provide a closedform Kaiser-like method for achieving prescribed specifications while minimizing the filter length $N$ through the appropriate selection of the window parameters. However, for some applications one may be willing to increase $N$ to achieve different frequency-selectivity characteristics. For instance, increased stopband rolloff, that is, increased suppression of stopband energy furthest from the transition bandwidth (see [20]), can be achieved by increasing parameter $\mu$ but this has the effect of decreasing the stopband attenuation. Thus to achieve the same stopband attenuation, $N$ must be increased. To accommodate these scenarios, estimates for $D$ and $\beta$ were obtained as

$$
\begin{aligned}
& D=a A_{a}^{2}+b A_{a}+c, \\
& \beta= \begin{cases}a_{1} A_{a}^{2}+b_{1} A_{a}+c_{1} & \text { for } A_{a} \leq 60, \\
a_{2} A_{a}^{2}+b_{2} A_{a}+c_{2} & \text { for } A_{a}>60\end{cases}
\end{aligned}
$$

for the values $\mu=\{0,0.1,0.2, \ldots, 1.0\}$, where the model coefficients are given in Tables 1 and 2, respectively. The estimate for $D$ should be used in conjunction with (33) to predict the required value of $N$ for the particular selection of $\mu$ and a set of prescribed filter specifications. Estimates for $D$ and $\beta$ that correspond to values of $\mu$ in the range $[0,1]$ that are not included in Tables 1 and 2 can be obtained using cubic spline interpolation, where $\left(\mu_{i}\right)_{1}^{n}=\{0,0.1,0.2, \ldots, 1.0\}$ are the abscissa values and $\left(D_{i}\right)_{1}^{n}$ and $\left(\beta_{i}\right)_{1}^{n}$ are their corresponding ordinate values (see [21, Chapter 7]). This window-parameter alteration technique can provide designers with a simple approach for tailoring a filter's frequency selectivity for a particular application while still achieving prescribed specifications. 
TABLE 2: Model coefficients for parameter $\beta$.

\begin{tabular}{|c|c|c|c|c|c|c|}
\hline$\mu$ & $a_{1}$ & $b_{1}$ & $c_{1}$ & $a_{2}$ & $b_{2}$ & $c_{2}$ \\
\hline 0.0 & $7.337 \mathrm{E}-5$ & $2.533 \mathrm{E}-2$ & $3.404 \mathrm{E}-1$ & $1.534 \mathrm{E}-5$ & $3.183 \mathrm{E}-2$ & $1.585 \mathrm{E}-1$ \\
\hline 0.1 & $7.895 \mathrm{E}-5$ & $2.430 \mathrm{E}-2$ & $3.401 \mathrm{E}-1$ & $1.680 \mathrm{E}-5$ & $3.142 \mathrm{E}-2$ & $1.357 \mathrm{E}-1$ \\
\hline 0.2 & $8.930 \mathrm{E}-5$ & $2.265 \mathrm{E}-2$ & $3.645 \mathrm{E}-1$ & $1.811 \mathrm{E}-5$ & $3.094 \mathrm{E}-2$ & $1.233 \mathrm{E}-1$ \\
\hline 0.3 & $1.126 \mathrm{E}-4$ & $1.971 \mathrm{E}-2$ & $4.261 \mathrm{E}-1$ & $1.847 \mathrm{E}-5$ & $3.055 \mathrm{E}-2$ & $1.126 \mathrm{E}-1$ \\
\hline 0.4 & $1.240 \mathrm{E}-4$ & $1.774 \mathrm{E}-2$ & $4.779 \mathrm{E}-1$ & $2.434 \mathrm{E}-5$ & $2.912 \mathrm{E}-2$ & $1.535 \mathrm{E}-1$ \\
\hline 0.5 & $1.265 \mathrm{E}-4$ & $1.656 \mathrm{E}-2$ & $5.203 \mathrm{E}-1$ & $5.085 \mathrm{E}-5$ & $2.439 \mathrm{E}-2$ & $3.260 \mathrm{E}-1$ \\
\hline 0.6 & $1.134 \mathrm{E}-4$ & $1.690 \mathrm{E}-2$ & $5.359 \mathrm{E}-1$ & $7.947 \mathrm{E}-5$ & $1.956 \mathrm{E}-2$ & $5.033 \mathrm{E}-1$ \\
\hline 0.7 & $8.981 \mathrm{E}-5$ & $1.845 \mathrm{E}-2$ & $5.281 \mathrm{E}-1$ & $7.299 \mathrm{E}-5$ & $2.120 \mathrm{E}-2$ & $4.171 \mathrm{E}-1$ \\
\hline 0.8 & $6.355 \mathrm{E}-5$ & $2.070 \mathrm{E}-2$ & $5.033 \mathrm{E}-1$ & $3.755 \mathrm{E}-5$ & $2.748 \mathrm{E}-2$ & $1.763 \mathrm{E}-1$ \\
\hline 0.9 & $8.045 \mathrm{E}-5$ & $1.987 \mathrm{E}-2$ & $5.308 \mathrm{E}-1$ & $2.149 \mathrm{E}-5$ & $2.983 \mathrm{E}-2$ & $1.373 \mathrm{E}-1$ \\
\hline 1.0 & $9.410 \mathrm{E}-5$ & $1.925 \mathrm{E}-2$ & $5.550 \mathrm{E}-1$ & $9.433 \mathrm{E}-6$ & $3.158 \mathrm{E}-2$ & $1.144 \mathrm{E}-1$ \\
\hline
\end{tabular}

\subsection{Design algorithm}

Based on the findings of the previous section, a lowpass nonrecursive filter that would satisfy the specifications

(i) passband edge: $\omega_{p}$,

(ii) stopband edge: $\omega_{a}$,

(iii) passband ripple: $A_{p}$,

(iv) stopband ripple: $A_{a}$,

(v) sampling frequency: $\omega_{s}$

can be designed using Algorithm 1.

\subsection{Comparison with other windows}

The performance of different windows was compared by designing filters for fixed values of $N$ and $\omega_{c}$ [3]. The transition bandwidth was measured for the resulting filters and used to calculate $D$ using (31). Figure 5 shows plots of the stopband attenuation versus $D$ for $N=127, \omega_{c}=0.4 \pi \mathrm{rad} / \mathrm{s}$, and $\omega_{s}=2 \pi \mathrm{rad} / \mathrm{s}$ for a variety of fixed and adjustable windows. Expressions for these windows can be found either in [10] or [22], while the Nuttall window is described in [23]. For the adjustable windows (Kaiser, Dolph-Chebyshev, Saramäki, ultraspherical, and Gaussian windows), a number of filters were designed by altering the independent window parameter. As can be seen, the ultraspherical window offers better performance than the Kaiser, Dolph-Chebyshev, Saramäki, and Gaussian windows achieving an average increase in the stopband attenuation of $2.48 \mathrm{~dB}$ relative to the Kaiser window, $4.29 \mathrm{~dB}$ relative to the Dolph-Chebyshev window, and $2.21 \mathrm{~dB}$ relative to the Saramäki window. The Gaussian window provides much poorer results than the other adjustable windows. For the sake of comparison, equiripple designs based on the weighted-Chebyshev method of ParksMcClellan [11] were also carried out assuming that $\delta_{p}=\delta_{a}$. The weighted-Chebyshev method increases the stopband attenuation by about $2.93 \mathrm{~dB}$ on the average but this is to be expected since the Remez algorithm yields designs that are $L_{\infty}$ optimal.

The performance of different windows was also compared by finding the required filter length to achieve a set of prescribed specifications. Figure 6 shows plots of the actual stopband attenuations achieved for a fixed transition
Step 1: Input $\omega_{p}, \omega_{a}, A_{p}, A_{a}$, and $\omega_{s}$. Find the "design" $\delta$ using (28) and then update $A_{a}$ using (29).

Step 2: Calculate the window parameter $\mu$ using (32).

Step 3: Calculate the filter length $N$ using (33) in conjunction with (30) and (34). Round $N$ up to the nearest odd integer.

Step 4: Calculate the window parameter $x_{\mu}$ using (7) in conjunction with (35) and the method described in [8] for calculating $x_{N-1}^{(\mu)}$.

Step 5: With $\mu, x_{\mu}$, and $N$ known, the coefficients of the ultraspherical window can be generated from (19).

Step 6: Calculate the relevant terms of the infinite-duration impulse response using (21) with $\omega_{c}=\left(\omega_{p}+\omega_{a}\right) / 2$.

Step 7: Obtain the noncausal finite-duration impulse response using (22).

Step 8: Obtain the causal design using (23).

Step 9: Check the design obtained to ensure that the filter satisfies the prescribed specifications. If it does not, increase $N$ by 2 and go to Step 4 .

Algorithm 1: Lowpass filter design using the ultraspherical window.

bandwidth of $B_{t}=0.2 \mathrm{rad} / \mathrm{s}$ and filter length $N$ for lowpass filters designed using the Kaiser, Dolph-Chebyshev, and ultraspherical windows. Results for the Saramäki window have been omitted as they are very similar to those of the Kaiser window. The filters were designed to achieve the transition bandwidth $B_{t}=0.2 \mathrm{rad} / \mathrm{s}$ to a high degree of precision by fine-tuning the independent window parameter using optimization techniques. As can be seen, for a given filter length, the ultraspherical window increases the stopband attenuation relative to the Kaiser and Dolph-Chebyshev windows achieving on the average an increase of $2.61 \mathrm{~dB}$ relative to the Kaiser window and $4.49 \mathrm{~dB}$ relative to the DolphChebyshev window. Alternatively, for prescribed specifications, the ultraspherical window yields lower-order filters than the Kaiser or Dolph-Chebyshev windows. On the other hand, the weighted-Chebyshev method increases the stopband attenuation relative to the ultraspherical window by about $2.76 \mathrm{~dB}$ on the average. 


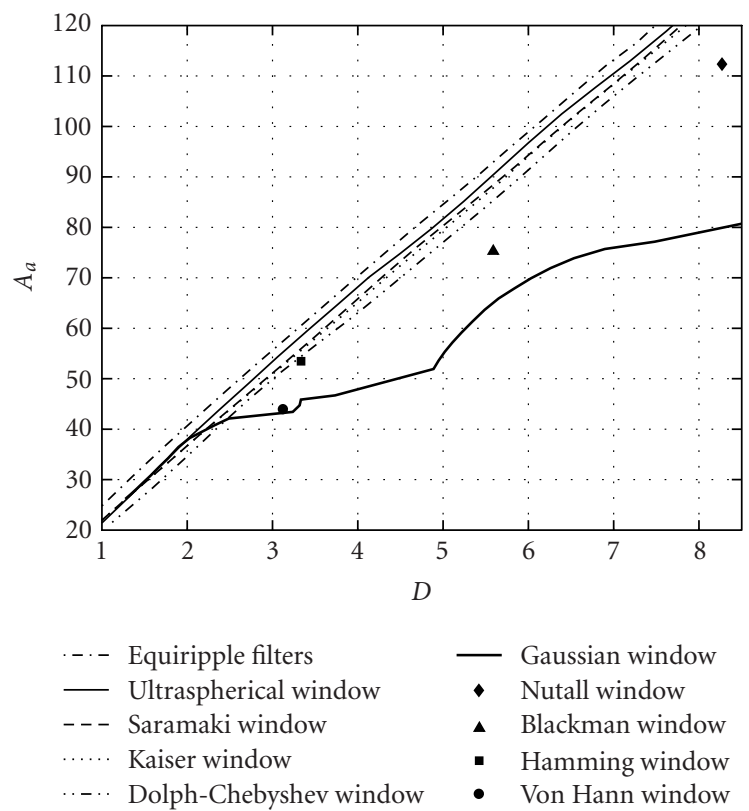

FIgURe 5: Stopband attenuation versus $D$ for filters designed using various windows with $N=127$ and $\omega_{c}=0.4 \pi$ rads/s. Results for equiripple filters of the same length with $\delta_{p}=\delta_{a}$ are included for comparison.

\subsection{Highpass, bandpass, and bandstop filters}

The above design method can be readily extended to the design of highpass, bandpass, and bandstop filters by following the procedure of [10]. For instance, the specifications for bandstop filters assume the form

$$
\begin{array}{rlrl}
1-\delta_{p} \leq H\left(e^{j \omega T}\right) & \leq 1+\delta_{p} & & \text { for }|\omega| \in\left[0, \omega_{p 1}\right], \\
-\delta_{a} \leq H\left(e^{j \omega T}\right) \leq \delta_{a} & & \text { for }|\omega| \in\left[\omega_{a 1}, \omega_{a 2}\right], \\
1-\delta_{p} \leq H\left(e^{j \omega T}\right) \leq 1+\delta_{p} & \text { for }|\omega| \in\left[\omega_{p 2}, \frac{\omega_{s}}{2}\right] .
\end{array}
$$

The ideal frequency response is taken as

$$
H_{i d}\left(e^{j \omega T}\right)= \begin{cases}1 & \text { for } 0 \leq|\omega|<\omega_{c 1} \\ 0 & \text { for } \omega_{c 1} \leq|\omega| \leq \omega_{c 2} \\ 1 & \text { for } \omega_{c 2}<|\omega| \leq \frac{\omega_{s}}{2}\end{cases}
$$

with

$$
\omega_{c 1}=\omega_{p 1}+\frac{B_{t}}{2}, \quad \omega_{c 2}=\omega_{p 2}-\frac{B_{t}}{2},
$$

where the design is based on the narrower of the two transition bandwidths, that is,

$$
B_{t}=\min \left[\left(\omega_{a 1}-\omega_{p 1}\right),\left(\omega_{p 2}-\omega_{a 2}\right)\right] .
$$

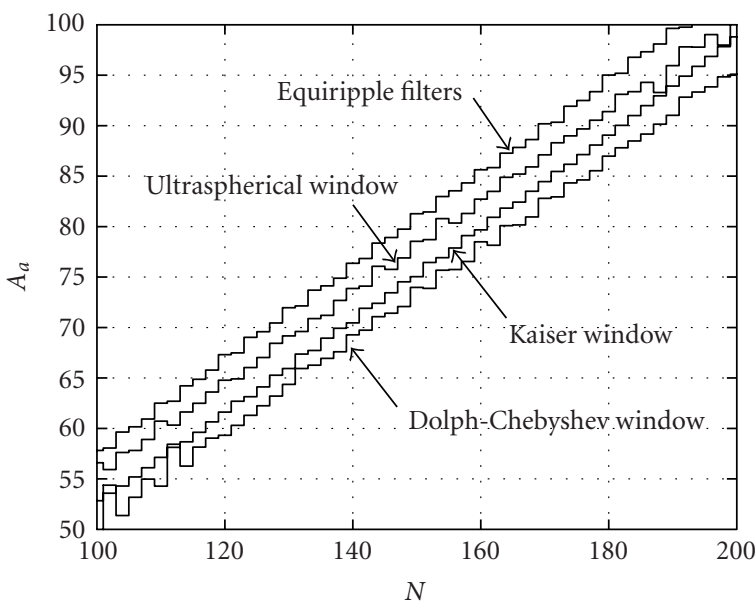

Figure 6: Actual stopband attenuation $A_{a}$ achieved by filters designed with length $N$ and transition bandwidth $B_{t}=0.2 \mathrm{rad} / \mathrm{s}$.

Straightforward analysis gives the infinite-duration impulse response as

$$
h_{i d}(n T)= \begin{cases}1-\frac{\left(\omega_{c 2}-\omega_{c 1}\right)}{\pi} & \text { for } n=0, \\ \frac{\sin \left(\omega_{c 1} n T\right)-\sin \left(\omega_{c 2} n T\right)}{n \pi} & \text { for } n \neq 0 .\end{cases}
$$

Using similar modifications [10], Algorithm 1 can be readily extended to the design of highpass and bandpass filters as well as multiband filters [24].

\section{DIGITAL DIFFERENTIATORS AND HILBERT TRANSFORMERS}

One advantage of the window method is the ease with which it can be applied to a wide range of filter design problems. In this section, we employ the window method for the design of digital differentiators and Hilbert transformers.

\subsection{Digital differentiators}

In signal processing, the need often arises for the derivative of a signal at some time instant $t=t_{1}$. For example, if $y(n T)$ is required to be the first derivative of $x(t)$ at $t=n T$, we can write

$$
y(n T)=f[x(t)]=\left.\frac{d x(t)}{d t}\right|_{t=n T} .
$$

Digital differentiators (DDs) have an ideal frequency response

$$
H\left(e^{j \omega T}\right)=j \omega \quad \text { for }|\omega| \leq \frac{\omega_{s}}{2} .
$$

In radar and sonar, object tracking can be accomplished using velocity and acceleration measurements calculated by applying differentiators to position data [25]. Differentiators are also used in plant control applications such as the PID controller [26], biomedical and geophysics signal processing, and image processing systems. Consequently, the design of differentiators is of considerable importance. 
TABle 3: Estimate coefficients for parameter $A_{\text {cor }}$.

\begin{tabular}{cccc}
\hline Window function & $a$ & $b$ & $c$ \\
\hline Kaiser & 2.422 & -13.73 & 10.86 \\
Dolph-Chebyshev & 2.700 & -14.23 & 12.25 \\
Ultraspherical & 1.506 & -11.10 & 8.170 \\
\hline
\end{tabular}

Since differentiators amplify high-frequency errors such as instrumentation measurement errors, bandlimited differentiators are quite useful. Practical bandlimited differentiator design can be accomplished in terms of a nonrecursive filter whose frequency response is required to satisfy the equations

$$
\begin{aligned}
j\left(\omega-\delta_{p}\right) \leq H\left(e^{j \omega T}\right) \leq j\left(\omega+\delta_{p}\right) & \text { for }|\omega| \in\left[0, \omega_{p}\right], \\
-j\left(\delta_{a}\right) \leq H\left(e^{j \omega T}\right) \leq j\left(\delta_{a}\right) & \text { for }|\omega| \in\left[\omega_{a}, \frac{\omega_{s}}{2}\right] .
\end{aligned}
$$

For a bandlimited differentiator, the ideal frequency response is taken as

$$
H_{i d}\left(e^{j \omega T}\right)= \begin{cases}j \omega & \text { for }|\omega| \leq \omega_{c} \\ 0 & \text { for } \omega_{c}<|\omega| \leq \frac{\omega_{s}}{2}\end{cases}
$$

with $\omega_{c}=\left(\omega_{p}+\omega_{a}\right) / 2$. Straightforward analysis gives the infinite-duration impulse response as

$$
h_{i d}(n T)= \begin{cases}\frac{\omega_{c} \cos \left(n \omega_{c}\right)}{n \pi}-\frac{\sin \left(n \omega_{c}\right)}{n^{2} \pi} & \text { for } n \neq 0, \\ 0 & \text { for } n=0 .\end{cases}
$$

The transition bandwidth in (33) is

$$
B_{t}=\omega_{a}-\omega_{p} .
$$

In DDs, the passband ripple and stopband attenuation are dependant on the cutoff frequency of the differentiator. To account for this, a correction for $A_{a}$ of the form

$$
A_{a}^{\prime}=A_{a}-A_{\text {cor }}
$$

is required where $A_{a}^{\prime}$ is the corrected design attenuation whose value replaces $A_{a}$ in Algorithm $1, A_{a}$ is the desired design attenuation, and $A_{\text {cor }}$ is a correction term given by

$$
A_{\mathrm{cor}}=a \omega_{c}^{2}+b \omega_{c}+c
$$

The values of the coefficients $a, b$, and $c$ for the Kaiser, Dolph-Chebyshev, and ultraspherical windows are given in Table 3. Examples of differentiators designed using the window method and Remez algorithm can be found in [10].

\subsection{Hilbert transformers}

In some digital signal processing applications, it is necessary to form a special version of the input signal $x(n T)$, designated $\tilde{x}(n T)$, with a frequency spectrum equal to that of $x(n T)$ for the positive Nyquist interval and zero for the negative Nyquist interval [10]. Signals of this type are referred to as analytic signals and can be generated by a complex filter with frequency response

$$
H_{A}\left(e^{j \omega T}\right)=1+j H\left(e^{j \omega T}\right),
$$

where $H\left(e^{j \omega T}\right)$ is a Hilbert transformer which has an ideal frequency response given by

$$
H\left(e^{j \omega T}\right)=\left\{\begin{aligned}
-j & \text { for } 0<\omega<\frac{\omega_{s}}{2}, \\
j & \text { for }-\frac{\omega_{s}}{2}<\omega<0 .
\end{aligned}\right.
$$

Filters that perform this operation find application in frequency-division multiplexing systems using singlesideband modulation. Hilbert transformers can be designed in terms of a nonrecursive filter whose frequency response is required to satisfy the equations

$$
\begin{gathered}
j\left(1-\delta_{p}\right) \leq H\left(e^{j \omega T}\right) \leq j\left(1+\delta_{p}\right) \quad \text { for } \omega \in\left[\omega_{p 1}, \frac{\omega_{s}}{2}\right], \\
j\left(-1-\delta_{p}\right) \leq H\left(e^{j \omega T}\right) \leq j\left(-1+\delta_{p}\right) \quad \text { for } \omega \in\left[-\frac{\omega_{s}}{2},-\omega_{p 1}\right] .
\end{gathered}
$$

Straightforward analysis gives the infinite-duration impulse response as

$$
h_{i d}(n T)= \begin{cases}\frac{2}{n \pi} \sin ^{2} \frac{n \pi}{2} & \text { for } n \neq 0 \\ 0 & \text { for } n=0\end{cases}
$$

The transition bandwidth in (33) is

$$
B_{t}=2 \omega_{p 1} .
$$

Like differentiators, it was found that Hilbert transformers require a correction for $A_{a}$ of the form

$$
A_{a}^{\prime}=A_{a}+A_{\text {cor }},
$$

where $A_{a}^{\prime}$ is the corrected design attenuation whose value replaces $A_{a}$ in Algorithm $1, A_{a}$ is the desired design attenuation in $\mathrm{dB}$, and $A_{\text {cor }}$ is a correction term given by $A_{\text {cor }}=$ 6.414, 5.236, and 6.457 for the Kaiser, Dolph-Chebyshev, and ultraspherical windows, respectively.

\section{EXAMPLES}

Example 1. Design a lowpass filter with $\omega_{p}=1, \omega_{a}=1.2 \mathrm{rad} / \mathrm{s}$, and $A_{a}=80 \mathrm{~dB}$ using the Kaiser, Dolph-Chebyshev, and ultraspherical windows.

The adjustable parameters for the Kaiser, DolphChebyshev, and ultraspherical windows were calculated as $\alpha=7.857, \beta=2.803$, and $\beta=2.574$, respectively. The additional parameter calculated for the ultraspherical window was $\mu=0.6173$. The stopband attenuations achieved were $79.38,82.27$, and $79.36 \mathrm{~dB}$ with transition bandwidths $0.1987,0.1994$, and $0.1965 \mathrm{rad} / \mathrm{s}$, respectively.

To achieve the desired specifications more precisely a simple technique described in [1] can be employed. First, the actual stopband attenuation of the filter $A_{a r}$ is measured for 
the estimated value of $\beta$. Then $\beta$ is reestimated using an adjusted design attenuation $A_{a}^{\prime}=A_{a}-\left(A_{a r}-A_{a}\right)$ where $A_{a}$ is the desired design stopband attenuation. With this modification, the recalculated parameters assume the values $\alpha=7.926$, $\beta=2.725$, and $\beta=2.596$, respectively. The stopband attenuations were $80.03,79.96$, and $79.83 \mathrm{~dB}$ with transition bandwidths $0.2005,0.1923$, and $0.1983 \mathrm{rad} / \mathrm{s}$, respectively. The filter lengths required to achieve the specifications were $N=159$ for the Kaiser window, $N=165$ for the DolphChebyshev window, and $N=153$ for the ultraspherical window. Figure 7 shows the amplitude responses of the designed filters.

Example 2. Design a bandstop filter with $\omega_{p 1}=0.5, \omega_{a 1}=$ 0.7, $\omega_{a 2}=2.0, \omega_{p 2}=2.2 \mathrm{rad} / \mathrm{s}$, and $A_{a}=40 \mathrm{~dB}$ using the Kaiser, Dolph-Chebyshev, and ultraspherical windows.

The adjustable parameters for the Kaiser, DolphChebyshev, and ultraspherical windows were calculated as $\alpha=3.395, \beta=1.471$, and $\beta=1.391$, respectively. The additional parameter calculated for the ultraspherical window was $\mu=0.5960$. The stopband attenuations achieved were $41.34,37.36$, and $38.40 \mathrm{~dB}$ with transition bandwidths $0.1963,0.2027$, and $0.2033 \mathrm{rad} / \mathrm{s}$, respectively. Using the modification discussed in Example 1 to improve stopband attenuation accuracy, the recalculated parameters assume the values $\alpha=3.235, \beta=1.554$, and $\beta=1.420$, respectively. The stopband attenuations were $39.92,40.07$, and $39.94 \mathrm{~dB}$ with transition bandwidths $0.1905,0.2188$, and $0.2125 \mathrm{rad} / \mathrm{s}$, respectively. The filter lengths required to achieve the specifications were $N=73$ for the Kaiser window, $N=73$ for the Dolph-Chebyshev window, and $N=67$ for the ultraspherical window. Figure 8 shows the amplitude responses of the designed filters.

Example 3. Design a Hilbert transformer with $\omega_{p 1}=$ $0.2 \mathrm{rad} / \mathrm{s}$ and $A_{p}=80 \mathrm{~dB}$ using the Kaiser, Dolph-Chebyshev, and ultraspherical windows.

The adjusted design attenuations from (55) for the Kaiser, Dolph-Chebyshev, and ultraspherical windows were calculated as $A_{a}^{\prime}=86.41,85.24$, and 86.46 , respectively. The adjustable parameters were calculated as $\alpha=8.564, \beta=$ 2.983 , and $\beta=2.789$, respectively, while the additional parameter for the ultraspherical window was calculated as $\mu=$ 0.6445 . The design attenuations achieved were $80.12,79.59$, and $79.39 \mathrm{~dB}$ with transition bandwidths $0.3941,0.3889$, and $0.3847 \mathrm{rad} / \mathrm{s}$, respectively. Using the modification discussed in Example 1 to improve design attenuation accuracy, the recalculated parameters assume the values $\alpha=$ $8.550, \beta=2.997$, and $\beta=2.809$, respectively. The attenuations were $79.96,80.01$, and $79.93 \mathrm{~dB}$ with transition bandwidths $0.3935,0.3912$, and $0.3879 \mathrm{rad} / \mathrm{s}$, respectively. The filter lengths required to achieve the specifications were $N=88$ for the Kaiser window, $N=90$ for the Dolph-Chebyshev window, and $N=86$ for the ultraspherical window. Figure 9 shows the amplitude responses of the designed Hilbert transformers.

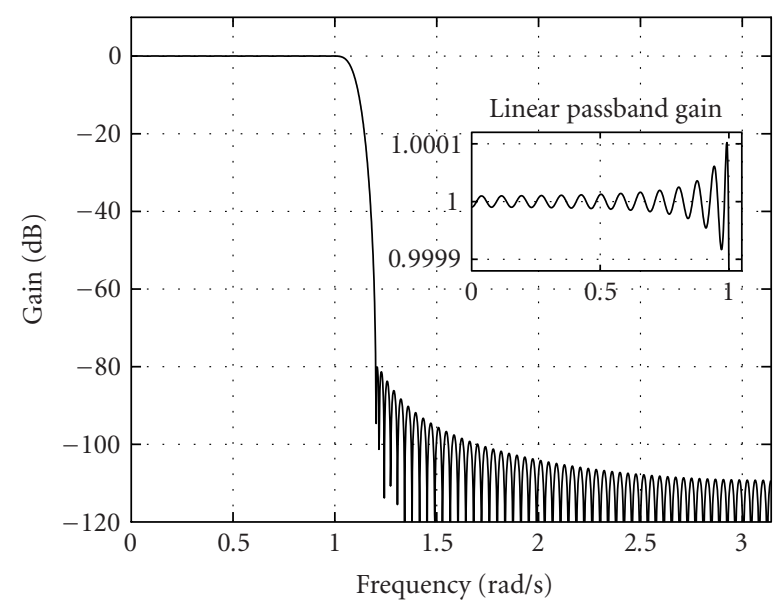

(a)

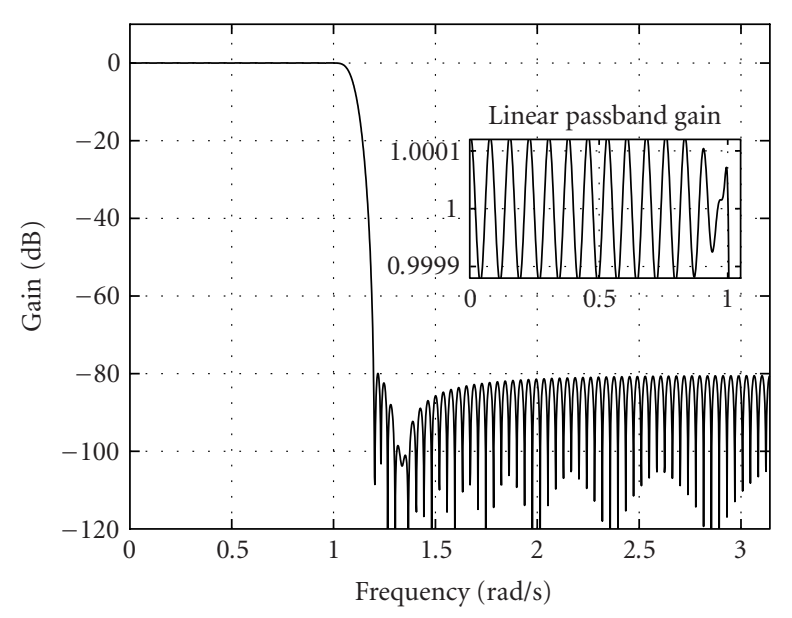

(b)

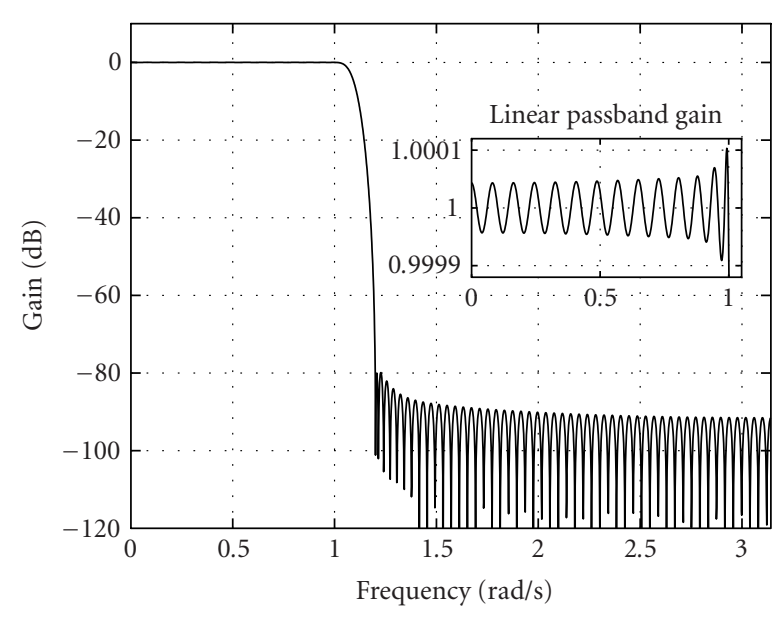

(c)

Figure 7: Example 1: amplitude responses of lowpass filters designed using various window functions. (a) Kaiser window. (b) Dolph-Chebyshev window. (c) Ultraspherical window. 


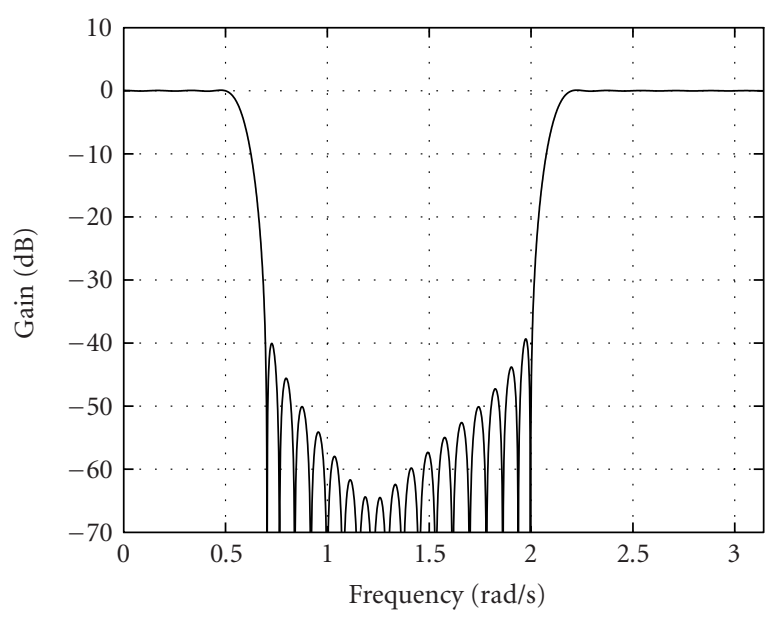

(a)

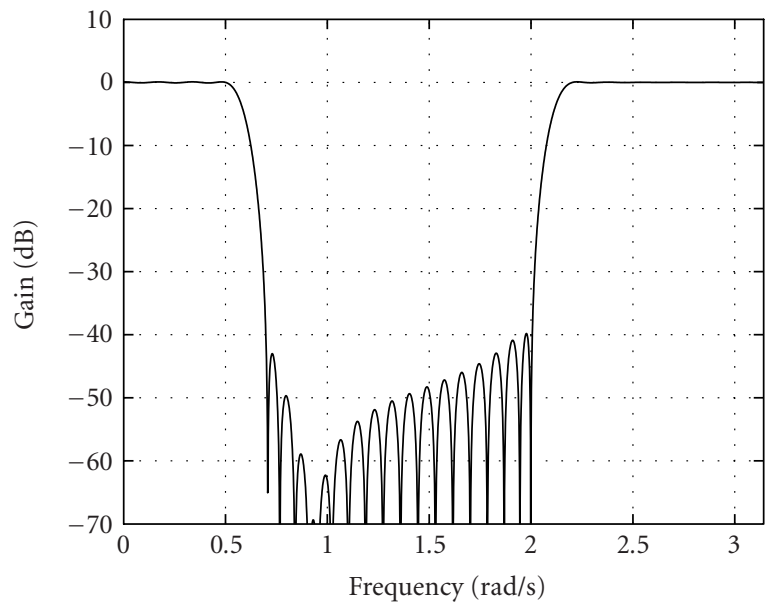

(b)

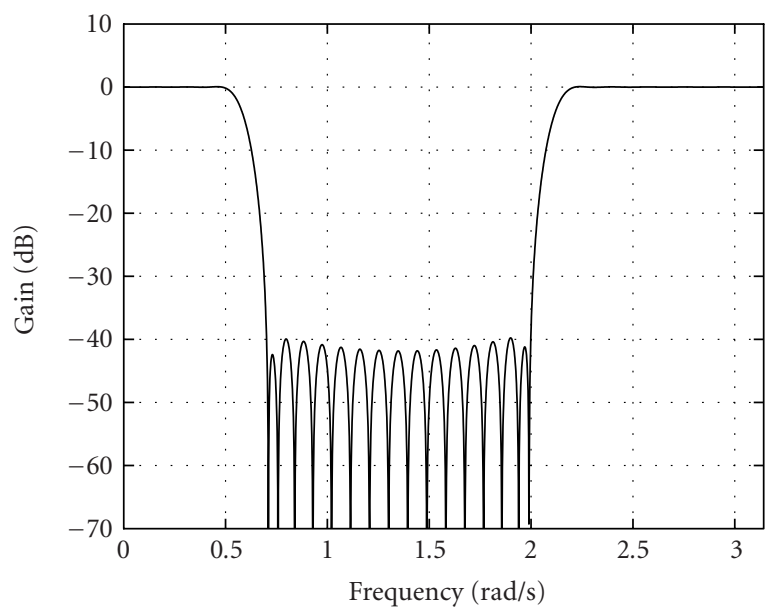

(c)

Figure 8: Example 2: amplitude responses of bandstop filters designed using various window functions. (a) Kaiser window. (b) Dolph-Chebyshev window. (c) Ultraspherical window.

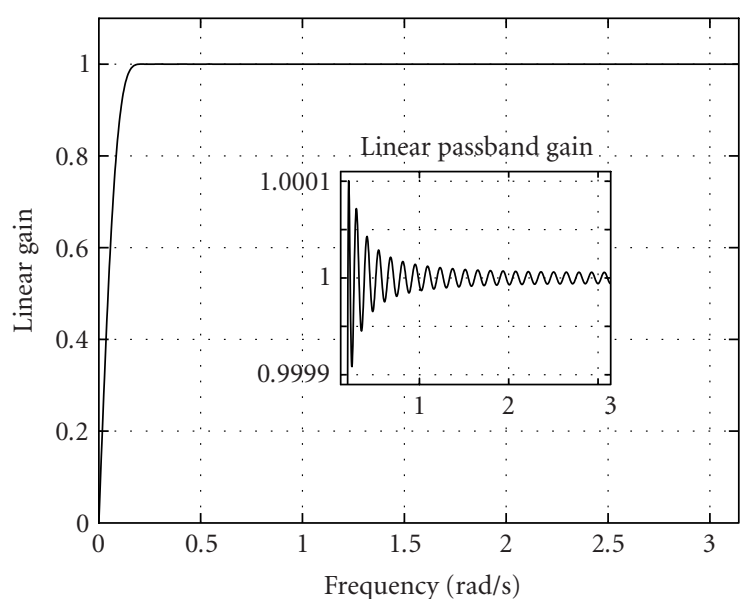

(a)

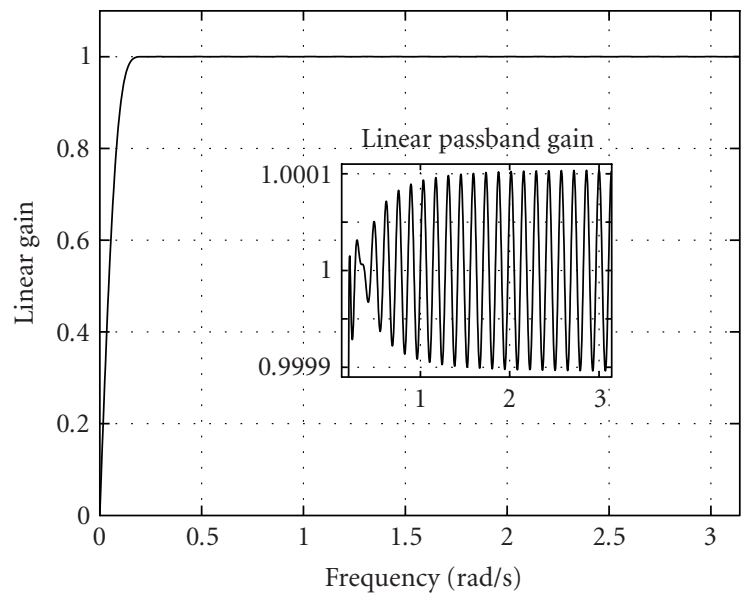

(b)

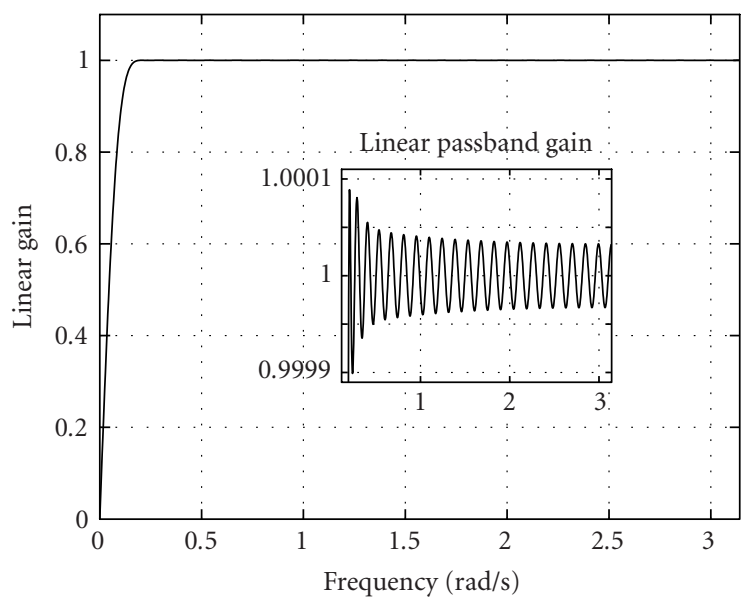

(c)

Figure 9: Example 3: amplitude responses of Hilbert transformers designed using various window functions. (a) Kaiser window. (b) Dolph-Chebyshev window. (c) Ultraspherical window. 


\section{CONCLUSIONS}

An efficient method for designing nonrecursive digital filters based on the ultraspherical window has been proposed. Economies in computation are achieved in two ways. First, through an efficient formulation of the window coefficients, the amount of computation required is reduced to a small fraction of that required by the standard methods. Second, the filter length and the independent window parameters that would be required to achieve prescribed specifications in lowpass, highpass, bandpass, and bandstop filters as well as in digital differentiators and Hilbert transformers are efficiently determined through empirical formulas. Experimental results indicate that the ultraspherical window yields lower order filters relative to designs obtained using a variety of other known windows including the Kaiser, Dolph-Chebyshev, and Saramäki windows. Alternatively, for a fixed filter length the ultraspherical window can provide reduced passband ripple and increased stopband attenuation relative to these windows. The weighted-Chebyshev method yields designs that are $L_{\infty}$ optimal but these designs require a large amount of computation, which makes them impractical for applications where the design has to be carried out online in real or quasireal time.

\section{REFERENCES}

[1] T. Saramäki, "Finite impulse response filter design," in Handbook for Digital Signal Processing, S. K. Mitra and J. F. Kaiser, Eds., Wiley \& Sons, New York, NY, USA, 1993.

[2] J. F. Kaiser, "Nonrecursive digital filter design using the $I_{0}$-sinh window function," in Proc. IEEE Int. Symp. Circuits and Systems (ISCAS '74), pp. 20-23, San Francisco, Calif, USA, April 1974.

[3] T. Saramäki, "A class of window functions with nearly minimum sidelobe energy for designing FIR filters," in Proc. IEEE Int. Symp. Circuits and Systems (ISCAS '89), vol. 1, pp. 359362, Portland, Ore, USA, May 1989.

[4] C. L. Dolph, "A current distribution for broadside arrays which optimizes the relationship between beamwidth and side-lobe level," Proc. IRE, vol. 34, pp. 335-348, 1946.

[5] R. Streit, "A two-parameter family of weights for nonrecursive digital filters and antennas," IEEE Trans. Acoust., Speech, Signal Processing, vol. 32, no. 1, pp. 108-118, 1984.

[6] A. G. Deczky, "Unispherical windows," in Proc. IEEE Int. Symp. Circuits and Systems (ISCAS '01), vol. 2, pp. 85-88, Sydney, NSW, Australia, May 2001.

[7] S. W. A. Bergen and A. Antoniou, "Generation of ultraspherical window functions," in Proc. 11th European Signal Processing Conference (EUSIPCO '02), vol. 2, pp. 607-610, Toulouse, France, September 2002.

[8] S. W. A. Bergen and A. Antoniou, "Design of ultraspherical window functions with prescribed spectral characteristics," EURASIP Journal on Applied Signal Processing, vol. 2004, no. 13, pp. 2053-2065, 2004.

[9] D. Johnson and J. Johnson, "Low-pass filters using ultraspherical polynomials," IEEE Trans. Circuits and Systems, vol. 13, no. 4, pp. 364-369, 1966.

[10] A. Antoniou, Digital Signal Processing: Signals, Systems, and Filters, McGraw-Hill, New York, NY, USA, 2005.

[11] T. Parks and J. McClellan, "Chebyshev approximation for nonrecursive digital filters with linear phase," IEEE Trans. Circuits and Systems, vol. 19, no. 2, pp. 189-194, 1972.
[12] J. McClellan and T. Parks, "A united approach to the design of optimum FIR linear-phase digital filters," IEEE Trans. Circuits and Systems, vol. 20, no. 6, pp. 697-701, 1973.

[13] D. J. Shpak and A. Antoniou, "A generalized Remez method for the design of FIR digital filters," IEEE Trans. Circuits and Systems, vol. 37, no. 2, pp. 161-174, 1990.

[14] S. H. Nawab, A. V. Oppenheim, A. P. Chandrakasan, J. M. Winograd, and J. T. Ludwig, "Approximate signal processing," Journal of VLSI Signal Processing Systems, vol. 15, no. 1-2, pp. 177-200, 1997.

[15] J. T. Ludwig, S. H. Nawab, and A. P. Chandrakasan, "Lowpower digital filtering using approximate processing," IEEE J. Solid-State Circuits, vol. 31, no. 3, pp. 395-400, 1996.

[16] C. J. Pan, "A stereo audio chip using approximate processing for decimation and interpolation filters," IEEE J. Solid-State Circuits, vol. 35, no. 1, pp. 45-55, 2000.

[17] M. Abramowitz and I. A. Stegun, Eds., Handbook of Mathematical Functions, vol. 55 of National Bureau of Standards Applied Mathematics Series, US Government Printing Office, Washington, DC, USA, 1964.

[18] L. R. Rabiner, J. F. Kaiser, O. Herrmann, and M. T. Dolan, "Some comparisons between FIR and IIR digital filters," Bell System Technical Journal, vol. 53, no. 2, pp. 305-331, 1974.

[19] O. Herrmann, L. R. Rabiner, and D. S. K. Chan, "Practical design rules for optimum finite impulse response low-pass digital filters," Bell System Technical Journal, vol. 52, no. 6, pp. 769-799, 1973.

[20] J. W. Adams, "FIR digital filters with least-squares stopbands subject to peak-gain constraints," IEEE Trans. Circuits and Systems, vol. 38, no. 4, pp. 376-388, 1991.

[21] W. Cheney and D. Kincaid, Numerical Mathematics and Computing, Brooks/Cole, Pacific Grove, Calif, USA, 1995.

[22] F. J. Harris, "On the use of windows for harmonic analysis with the discrete Fourier transform," Proc. IEEE, vol. 66, no. 1, pp. 51-83, 1978.

[23] A. H. Nuttall, "Some windows with very good sidelobe behavior," IEEE Trans. Acoust., Speech, Signal Processing, vol. 29, no. 1, pp. 84-91, 1981.

[24] S. K. Mitra, Digital Signal Processing: A Computer-Based Approach, McGraw-Hill, New York, NY, USA, 1998.

[25] M. I. Skolnik, Ed., Radar Handbook, McGraw-Hill, New York, NY, USA, 1990.

[26] K. Ogata, Ed., Modern Control Engineering, Prentice-Hall, Upper Saddle River, NJ, USA, 4th edition, 2002.

Stuart W. A. Bergen was born in Guildford, England, UK, on November 5, 1976. He received the B.S. degree in electrical engineering from the University of Calgary, Calgary, Alberta, Canada, in 1999. Currently, he is pursuing the Ph.D. degree in electrical engineering at the University of Victoria, Victoria, British Columbia, Canada. From 1997 to 1998 , he was a firmware/hardware designer at Wireless Matrix, Calgary, Alberta,

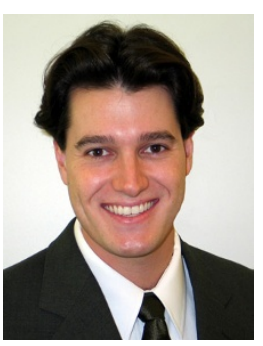
Canada, focusing on satellite telecommunications for the oil and gas industry. From 1998 to 2000, he was a firmware design engineer at Nortel Networks, Calgary, Alberta, Canada, concentrating on digital signal processing (DSP) for telecommunications systems. His research interests include DSP algorithms, digital filter design, multirate signal processing, beamforming, and bioinformatics. 
Andreas Antoniou received the B.S. (Engineering Honors) and Ph.D. degrees in electrical engineering from the University of London in 1963 and 1966, respectively, and is a Fellow of the IEE and IEEE. He served as the Founding Chair of the Department of Electrical and Computer Engineering, University of Victoria, Victoria, British Columbia, Canada, from July 1, 1983, to June 30, 1990, and is now Professor Emer-

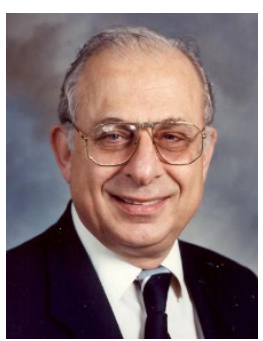

itus. His teaching and research interests are in the area of digital signal processing. He is the author of Digital Signal Processing: Signals, Systems, and Filters to be published by McGraw-Hill in the near future. Dr. Antoniou served as an Associate Editor of the IEEE Transactions on Circuits and Systems from June 1983 to May 1985 and as an Editor from June 1985 to May 1987, as a Distinguished Lecturer of the IEEE Signal Processing Society in 2003, and as the General Chair of the 2004 International Symposium on Circuits and Systems. He was awarded Ambrose Fleming Premium of 1964 by the IEE (Best Paper Award), a CAS Golden Jubilee Medal from the IEEE Circuits and Systems Society, the B.C. Science Council Chairman's Award for Career Achievement for 2000, the Doctor Honoris Causa degree by the Metsovio National Technical University, Athens, Greece, in 2002, and the IEEE Circuits and Systems Society Technical Achievements Award for 2005. 